\title{
SOIL ATTRIBUTES AND WEED SEEDBANK SPATIAL CORRELATION
}

\author{
CORRELAÇÃO ESPACIAL ENTRE ATRIBUTOS FÍSICOS E QUÍMICOS DO SOLO E \\ O BANCO DE SEMENTES DE PLANTAS INFESTANTES
}

\section{Ígor Araujo Menezes de AVILA ${ }^{1}$; Sandro Manuel Carmelino HURTADO Gabriel Camargo de JEZUS ${ }^{3}$; Gustavo Costa SILVA ${ }^{1}$; Matheus Mendes REZENDE ${ }^{1}$}

1. Graduando em Agronomia, Instituto de Ciências Agrárias - ICIAG, Universidade Federal de Uberlândia - UFU, Uberlândia, MG, Brasil. igoramnz@gmail.com@gmail.com; 2. Professor, Doutor, Instituto de Ciências Agrárias - ICIAG, UFU, Uberlândia, MG, Brasil; 3. Graduando em Agronomia, Instituto Federal do Triangulo Mineiro - IFTM, Uberlândia, MG, Brasil.

\begin{abstract}
The crop yield potential is affected by the crop-weed competition and their control create a dependence on herbicide use who brings, as consequence, soil impacts. Knowing the weed's spatial distribution on the field is a feasible alternative for improving the crop yield. The goal of this paper is the identification of the spatial variability on physical and chemical attributes of soil as well as the weed's seedbank so that, when correlated, may find standards to help on field management. The experiment was conducted on Uberlandia Federal University premises at soybean no-till area. Using georeferenced soil samples, were analyzed the physical and chemical attributes as well as the weed's seedbank. The weed population on controlled environment was quantify, sorting out broadleaf and grassy weeds species. The obtained data were analyzed by descriptive statistic and geostatistics for a semivariogram modeling, interpolation by the kriging methodology and the spatial variability maps achievement. The average value, coefficient of variation (CV\%), asymmetry, kurtosis coefficient and the significant linear correlations interfered on data spatial variability which we concluded by the spatial dependences on the attributes that had a linear correlation between them. The semivariograms presented varied range between 202 to 752 meters. Using the maps, verified two different regions for the broadleaf and grassy weeds seedbank. For both situations there was influence by the soil attributes on infestation level, which makes it possible to target the herbicide management reducing costs and the environmental impact. From the analyzed data we conclude that there is a spatial dependence for the physical and chemical soil attributes and their spatial distribution explains the weed seedbank spatial variability.
\end{abstract}

KEYWORDS: Precision Agriculture. Soil fertility. Spatial variability.

\section{INTRODUCTION}

The Brazil is recognized by its agricultural potential, where the soybean (Glycine max L.) stands out with productive levels close to 120 million tons per year (CONAB, 2018). However, the soybean genetic potential may be reduced by biotic and abiotic factors as crop-weed competition (VASCONCELOS et al., 2012), which changes the crop morphophysiology (LAMEGO et al., 2005), besides the additional costs for the weed control. Silva et al. (2009) related a dry mass accumulation reduction and a leaf area index modification. Formigheiri et al. (2018) showed a soybean seeds vigor and germination index reduction by studying the allelopathic influence of Ambrosia artemisiifolia.

According to IMEA (2018), the chemical weed control represented $4 \%$ of the total production cost per hectare in 2017. For this reason, the watercourses contamination by agrochemicals it is a constant concern to ecosystems (URI et al., 1998;
HOLLAND, 2004), where the residual effect achieves the main role on soil environment impact. On silty loam fields, Silva et al., (2011) observed phytotoxicity and crop biomass reduction because the residual effect of herbicides managed right before the crop planting.

According to Barroso el al., (2004), the negative impact reduction is obtained targeting the management at the specific areas under attack by weeds as well as by the herbicide application rates adjustment. Here it is necessary the knowledge on the weed's spatial distribution by identifying they canopy or seedbank (MONQUERO et al., 2008; IZQUIERDO et al., 2009). The qualitative and quantitative estimative is obtained by the direct germination of the soil samples or by the physical extraction of the seeds, associated to variability tests (LUSCHEI et al., 1998; MONQUERO; CHRISTOFFOLETTI, 2005).

The soil is subjected to chemical applications and shows heterogeneity and spatial distribution on its physical and chemical attributes 
(VIEIRA, 2000). It receives influence from the management system and its use (SOARES et al., 2011) also interferes on crop development, as reported by Shiratsuchi et al., (2005), on the high correlation between $\mathrm{pH}, \mathrm{Ca}, \mathrm{V} \%, \mathrm{~m} \%$ and the weed's seedbank.

To recognize the soil attributes and weed seedbank spatial variability (CLAY et al., 1999; JURADO-EXPOSITÓRIO，2003; SCHAFFRATH et al., 2007; CHIBA et al., 2010) it is a feasible alternative for reduce the production costs targeting the applications right on the observed parches as well as reducing the necessity on producing new maps because the temporal and spatial infestation stability (BARROSO et al., 2006; LONGCHAMPS et al., 2012).

The goal of this study was to identify the spatial variability on physical and chemical attributes of soil, as well as the weed seedbank so that, when correlated, we may find standards that could help on field management.

\section{MATERIAL AND METHODS}

The experiment was conducted on experimental farm Capim Branco (18 $53^{\circ}$ 'S e $48^{\circ} 20^{\prime} \mathrm{W}$ ), placed in Uberlandia/MG which belongs to Federal University of Uberlandia. The experimental field, which is 25 hectares wide, has the soil classified as a Red Latosol (EMBRAPA, 2006), soft wavy relief and Aw climate, according to Koppen classification. This study area is destined to no-till soybean growing since 2012 in succession planting with corn or sorghum.

The chemical (TEIXEIRA et al., 2017) and physical (CAMARGO et al., 2009) soil attributes evaluation was realized using 0 to $0,2 \mathrm{~m}$ deep georeferenced soil samples according to a dense and systemically randomized sampling grid two sampling points per hectare. These sampling points were about $75 \mathrm{~m}$ away. For each of the 50 grid points were randomly sampled 10 sub-samples with the aim of composing a composite sample.

The weed seedbank determination was obtained by sampling 3 sub-samples to compose a composite sample on the same 50 points, being them also 0 to $0,2 \mathrm{~m}$ deep. In order to better represent the weed seedbank, the soil samples were not sifted. After sampling, the soil samples were placed on $1,43 \mathrm{dm}^{3}$ bulk styrofoam trays under controlled environment. The moisture was monitored by daily irrigation aiming to offer the necessary conditions to seed's germination and emergency. Evaluations have done twice a week for a period of 14 weeks (MONQUEIRO;
CHRISTFFOLETI, 2005), sorting out broadleaf (B) and grassy $(\mathrm{G})$ weeds species.

The data were analyzed descriptively to obtain the average, minimum and maximum values as well as the coefficient of variation, asymmetry and kurtosis coefficient using the Sisvar software (FERREIRA, 2008) and Statistica software (STATSOFT, 2004). The geostatistical analysis, realized with the GS+ software (ROBERTSON, 1998), allowed the experimental semivariograms modelling (VIEIRA, 2000) and the interpolation by the kriging methodology for the spatial distribution maps obtainment.

\section{RESULTS}

The grassy weeds seedbank predominates over broadleaf weeds seedbank (Table 1). The grassy (8.2) and broadleaf (5.6) averages, away from the maximum value found for each evaluation (47 and 55, respectively) allow us to infer a possible spatial dependence presence for both groups of weeds (Table 1). The information can even be testified by the high $\mathrm{CV} \%$ values (WARRICK; NIELSEN, 1980) and by asymmetry and kurtosis coefficient found for both groups (Table 1) which characterize counting data, where the average does not adequately represent the sample population (CHIBA et al., 2010). In practical ways, this behavior may indicate parches existence (SHIRATSUCHI et al., 2005) independently of the preparation system (SHAFFRATH et al., 2007). For the attributes $\mathrm{Ca}, \mathrm{Mg}$ and sand, the $\mathrm{CV} \%$ was considered medium $(12<\mathrm{CV} \%<62)$ or near the average (V\%). For the grassy weeds data, the correlation with the soil attributes under analysis allowed us the testification of significant and negative values for the attribute sand $(\mathrm{r}=-0,32)$. A significate correlation was also obtained between broadleaf weeds and the attribute $\mathrm{Ca}(\mathrm{r}=0,39), \mathrm{Mg}$ $(\mathrm{r}=0,51)$ and base saturation $(\mathrm{r}=0,38)$.

The present data refers to the attributes where we observed a significant correlation. The spatial dependence was verified for the attributes with a linear correlation presence, except by the V\% (Table 2). For these attributes the semivariograms were better modeled to exponential and gaussian models, whereas, the parameter range showed values between 202 and $752 \mathrm{~m}$. From the data, we can infer that there is a more homogenous distribution on broadleaf seedbank when compared to grassy, which is reflected in the presence of bigger and lower incident parches or spots for the first group. The GDE (CAMBARDELLA et al., 
1994), classified from moderated to strong, evidences a good data collection.

Table 1. Weeds seedbank and physical-chemical soil attributes data descriptive statistic and geostatistics analysis. Uberlandia, 2018.

\begin{tabular}{|c|c|c|c|c|c|c|}
\hline \multirow{2}{*}{ Attribute } & \multirow{2}{*}{ Minimum } & \multirow{2}{*}{ Maximum } & \multirow{2}{*}{ Average } & \multirow{2}{*}{ CV\% } & \multicolumn{2}{|c|}{ Coefficient of } \\
\hline & & & & & Asymmetry & Kurtosis \\
\hline $\begin{array}{l}\text { Grassy weeds } \\
\text { (plants / tray) }\end{array}$ & 0.00 & 47.00 & 8.20 & 103.48 & 2.25 & 7.79 \\
\hline $\begin{array}{l}\text { Broadleaf weeds } \\
\text { (plants / tray) }\end{array}$ & 0.00 & 55.00 & 5.66 & 144.18 & 4.84 & 28.08 \\
\hline Calcium $\left(\mathrm{cmol}_{\mathrm{d}} / \mathrm{dm}^{3}\right)$ & 1.90 & 5.00 & 3.32 & 18.56 & 0.11 & 0.55 \\
\hline Magnesium $\left(\mathrm{cmol}_{\mathrm{c}} / \mathrm{dm}^{3}\right)$ & 0.40 & 1.70 & 0.82 & 26.83 & 1.35 & 1.30 \\
\hline$V(\%)^{1}$ & 37.95 & 67.31 & 51.60 & 11.67 & 0.14 & 0.37 \\
\hline Sand $(g / k g)$ & 347.00 & 710.00 & 531.92 & 17.52 & 0.24 & -0.95 \\
\hline Attribute & Model & $\mathrm{C}_{0}{ }^{3}$ & $\mathrm{C}_{0}+\mathrm{C}^{4}$ & $a(m)^{5}$ & GDE $^{6}$ & $\mathbf{r}^{2}$ \\
\hline $\begin{array}{l}\text { Grassy weeds } \\
\text { (plants / tray) }\end{array}$ & Gaussian & 0.10 & 55.92 & 202 & high & 0.71 \\
\hline $\begin{array}{l}\text { Broadleaf weeds } \\
\text { (plants / tray) }\end{array}$ & Gaussian & 37.40 & 124.00 & 752 & medium & 0.74 \\
\hline Calcium $\left(\mathrm{cmol}_{\mathrm{c}} / \mathrm{dm}^{3}\right)$ & Exponencial & 0.28 & 0.59 & 559 & medium & 0.31 \\
\hline Magnesium $\left(\mathrm{cmol}_{\mathrm{c}} / \mathrm{dm}^{3}\right)$ & Exponencial & 0.04 & 0.11 & 243 & medium & 0.50 \\
\hline $\mathrm{V}(\%)^{1}$ & $\mathrm{EPP}^{2}$ & 37.94 & - & - & - & - \\
\hline sand $(\mathrm{g} / \mathrm{kg})$ & Gaussian & 10.00 & 9130.00 & 663 & high & 0.99 \\
\hline
\end{tabular}

From the interpolated maps (Figure 1, 2 and 3 ), there is an aggregate behavior (SHIRATSUCHI et al., 2005; SCHAFFRATH et al., 2007), testifying the information obtained by the descriptive statistics

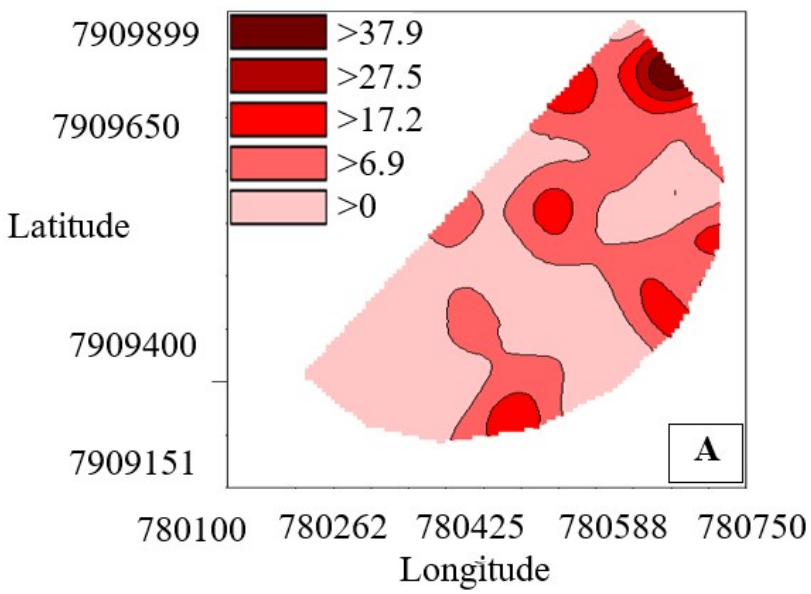

(Table 1). The grassy seedbank and the sand maps testified the negative correlation between them (Figure 1).

Figure 1. Interpolated maps by kriging methodology for the attributes A: grassy weeds (plants/tray) and B: sand $(\mathrm{g} / \mathrm{kg})$.

On field management, this information lights up and alert for the flat rate applications, considering, sometimes, the highest dose of the package insert recommendation. According to the data, lower doses can be applied on sandy areas as the southern region of the analyzed field (Figure
1B), inasmuch as these areas are prone to increased leaching indexes and herbicides availability (PROCÓPIO et al., 2001). Beyond the product saving possibility, there is a possible contaminations reduction, mainly in irrigated fields (FIRMINO et al., 2008). 

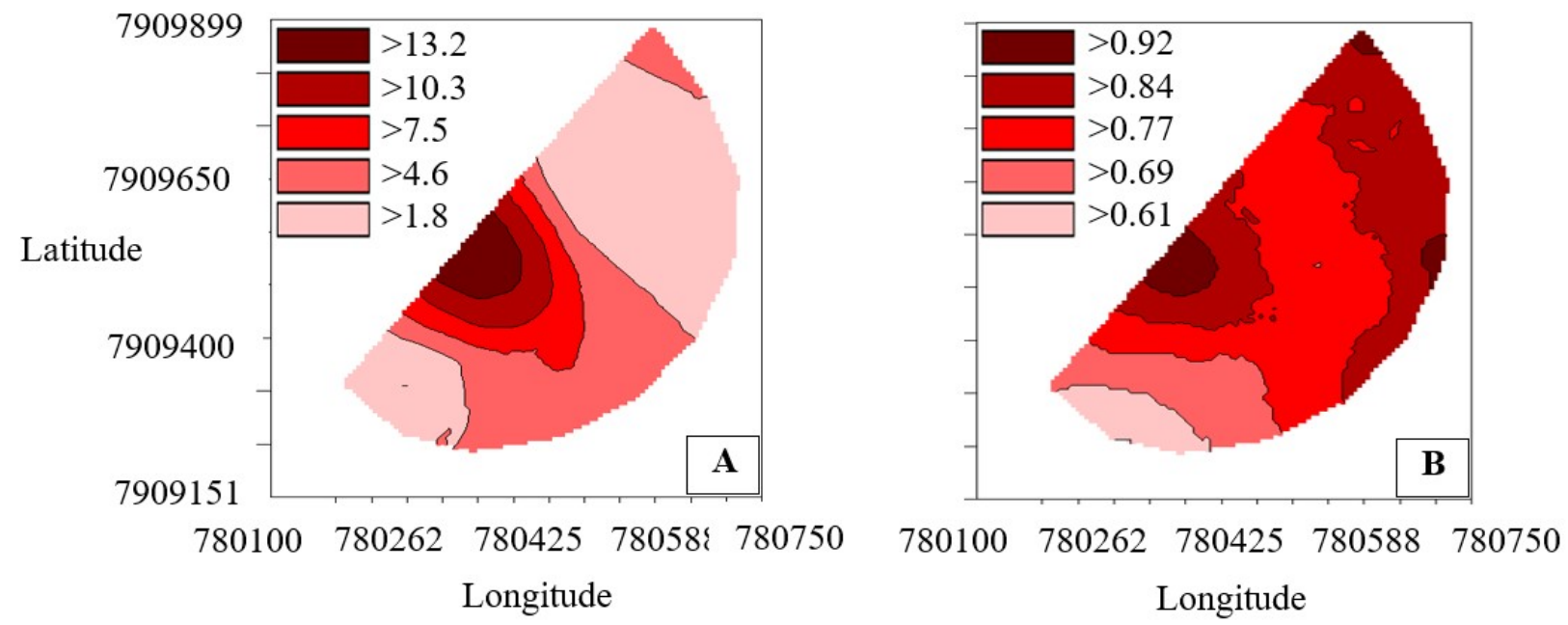

Figura 2. Interpolated maps by kriging methodology for the attributes A: broadleaf weeds (plants/tray) and B: magnesium $\left(\mathrm{cmolc} / \mathrm{dm}^{3}\right)$.
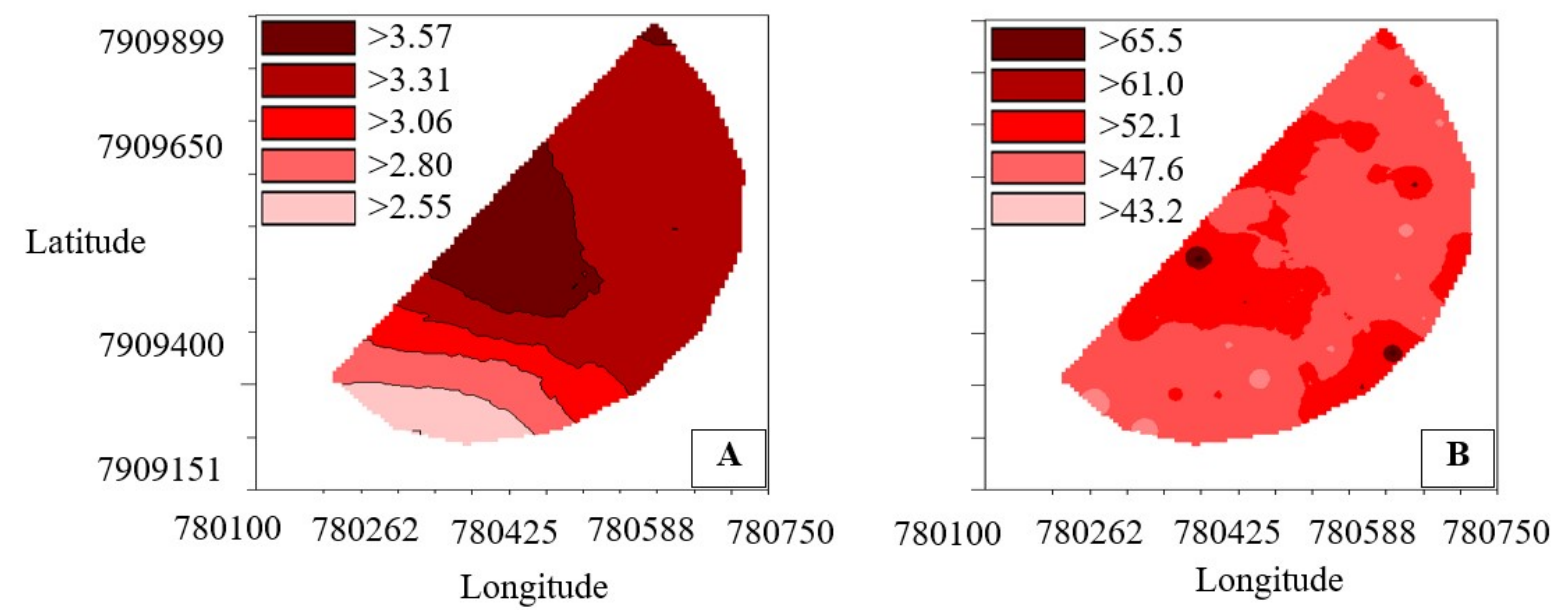

Figura 3. Interpolated maps by kriging methodology for the attributes $A$ : calcium $\left(\mathrm{cmolc} / \mathrm{dm}^{3}\right)$ and interpolated map by the inverse square distance for B: base saturation (\%).

According to the maps, the broadleaf seedbank parches spatial distribution may be justified by the soil fertility attributes distribution $\mathrm{Ca}, \mathrm{Mg} \% \mathrm{~V} \%$ (Figure 2A, 2B, 3A \& 3B), with linear correlations of $\mathrm{r}=0,39 ; \mathrm{r}=0,52 \& \mathrm{r}=0,38$, respectively. For both situations it is evidenced that the physical and chemical soil attributes contribute to the broadleaf and grassy weeds highest infestation levels.

Considering the large information amount available to this field, it becomes possible the definition of homogeneous zones or differentiated management units (CLAY et al., 1999; MOLIN et al., 2015), which allows the decision-making on herbicide doses management aiming for the costs and environment impacts reduction.

\section{CONCLUSION}

There is a spatial dependence for the physical and chemical soil attributes and their spatial distribution explains the weed seedbank spatial variability.

RESUMO: O potencial produtivo das culturas é afetado pela competição por plantas infestantes e o seu controle cria dependência no uso de herbicidas, com consequente impacto ao solo. Conhecer o comportamento espacial das espécies infestantes é alternativa viável no aumento do lucro das lavouras. O 
presente trabalho teve por objetivo identificar o comportamento espacial nos atributos físicos e químicos do solo e no banco de sementes de plantas infestantes, de maneira que, ao correlacioná-los possa-se encontrar padrões que auxiliem no manejo das áreas agrícolas. O estudo foi realizado em área pertencente à Universidade Federal de Uberlândia-MG em área cultivada com soja em plantio direto. A partir de amostragens georreferenciadas foi realizada avaliação química e física do solo e determinação do banco de sementes de plantas infestantes. Foi mensurado o número de plantas infestantes em ambiente controlado, separando as espécies germinadas em Folha Estreita e Folha Larga. Os dados foram avaliados pela estatística descritiva e geoestatística, para ajuste de semivariogramas, interpolação por krigagem e obtenção de mapas de distribuição espacial. Os valores de média, CV\%, assimetria, curtose, e as correlações lineares significativas inferiram no comportamento espacial dos dados, comportamento verificado pela dependência espacial observada para os atributos com correlação linear significativa entre eles. Os semivariogramas apresentaram alcance variando entre $202 \mathrm{~m}$ e $752 \mathrm{~m}$. A partir dos mapas verifica-se duas regiões distintas para o banco de plantas infestantes de folha estreita e larga. Para ambas situações houve contribuição dos atributos do solo nos níveis de infestação, o que possibilita o direcionamento no ajuste de doses herbicidas para redução de custos e impacto ambiental. A partir dos dados conclui-se que há comportamento espacial para atributos químicos e físicos de solo, e a sua distribuição espacial explica o comportamento espacial do banco de sementes de plantas infestantes.

PALAVRAS-CHAVE: Agricultura de precisão. Fertilidade do solo. Plantas daninhas.

\section{REFERENCES}

BARROSO, J., FERNÀNDEZ-QUINTANILLA, C., RUIZ, D., HERNAIZ, P., REW, L.J. Spatial stability of Avena sterilis ssp. ludoviciana populations under annual applications of low rates of imazamethabenz. Weed Research, v. 44, n. 3, p. 178-186, 2004. https://doi.org/10.1111/j.1365-3180.2004.00389.x

BARROSO, J., NAVARRETE, L., SÁNCHEZ DEL ARCO, M. J., FERNANDEZ-QUINTANILLA, C., LUTMAN, P. J. W., PERRY, N. H., HULL, R. I. Dispersal of Avena fatua and Avena sterilis patches by natural dissemination, soil tillage and combine harvesters. Weed Research, v. 46, n. 2, p. 118-128, 2006. https://doi.org/10.1111/j.1365-3180.2006.00500.x

CAMARGO, O.A., MONIZ, A.C., JORGE, J.A., VALADARES, J.M.A.S. Métodos de Análise Química, Mineralógica e Física de Solos do Instituto Agronômico de Campinas. Campinas: Instituto Agronômico, 2009.

CAMBARDELlA, C. A., MOORMAN, T. B., PARKIN, T. B., KARLEN, D. L., NOVAK, J. M., TURCO, R. F., KONOPKA, A. E. Field-scale variability of soil properties in central lowa soils. Soil Science Society of America Journal, v. 58, n. 5, p. 1501-1511, 1994. https://doi.org/10.2136/sssaj1994.03615995005800050033x

CHIBA, M.K., GUEDES FILHO, O., VIEIRA, S.R. Variabilidade espacial e temporal de plantas daninhas em Latossolo Vermelho argiloso sob semeadura direta. Acta Scientiarum-Agronomy, v. 32, n. 4, p. 735-742, 2010. https://doi.org/10.4025/actasciagron.v32i4.5445

CLAY, S.A., LEMS, G.J., CLAY, D.E., FORCELLA, F. Sampling weed spatial variability on a fieldwide scale. Weed Science, v. 47, n. 6, p. 674-681, 1999. https://doi.org/10.1017/S0043174500091323

CONAB. Companhia Nacional de Abastecimento. Acompanhamento da safra brasileira: grãos. quarto levantamento, janeiro, 2019. Disponível em: $<$ https://www.conab.gov.br/info-agro/safras/graos/boletim-dasafra-de-graos $>$. Acesso em 21 de janeiro de 2019.

EMBRAPA. EMPRESA BRASILEIRA DE PESQUISA AGROPECUÁRIA. Centro Nacional de Pesquisa de Solos. Sistema Brasileiro de Classificação de Solos. Rio de Janeiro: EMBRAPA-SPI, 2006. 
FERREIRA, D.F. Sisvar: um programa para análise e ensino de estatística. Revista Symposium, v. 6, n.1, p. 36-41, 2008.

FIRMINO, L.E., TUFFI SANTOS, L.D., FERREIRA, L.R., FERREIRA, F.A., QUIRINO, A.L.S. Movimento do herbicida imazapyr no perfil de solos tropicais. Planta Daninha, v. 26, n. 1, p. 223-230, 2008.

https://doi.org/10.1590/S0100-83582008000100023

FORMIGHEIRI, F.B., BONOME, L. T. S., BITTENCOURT, H. V. H., LEITE, K., REGINATTO, M., GIOVANETTI, L. K. Alelopatia de Ambrosia artemisiifolia na germinação e no crescimento de plântulas de milho e soja. Revista de Ciências Agrárias, v. 41, n. 3, p. 729-739, 2018. https://doi.org/10.19084/RCA18074

HOLLAND, J. M. The environmental consequences of adopting conservation tillage in Europe: reviewing the evidence. Agriculture, Ecosystems \& Environment, v. 103, n. 1, p. 1-25, 2004.

https://doi.org/10.1016/j.agee.2003.12.018

IMEA. Instituto Mato-grossense de Economia Agropecuaria. Boletim semanal da soja. janeiro, 2019.

Disponível em: $<\mathrm{http} / / \mathrm{www}$.imea.com.br/imea-site/relatorios-mercado-detalhe?c=4\&s=2>. Acesso em $21 \mathrm{de}$ janeiro de 2019.

IZQUIERDO, J., BLANCO-MORENO, J. M., CHAMORRO, L., GONZÁLEZ-ANDÚJAR, J. L., SANS, F. X, Spatial distribution of weed diversity within a cereal field. Agronomy for Sustainable Development, v. 29, n. 3, p. 491-496, 2009. https://doi.org/10.1051/agro/2009009

JURADO-EXPÓSITO, M.; LÓPEZ-GRANADOS, F.; GARCÍA-TORRES, L., GARCÍA-FERRER, A. Multispecies weed spatial variability and site-specific management maps in cultivated sunflower. Weed Science, v. 51, n. 3, p. 319-328, 2003. https://doi.org/10.1614/0043-1745(2003)051[0319:MWSVAS]2.0.CO;2

LAMEGO, F. P., FLECK, N. G., BIANCHI, M. A., VIDAL, R.A. Tolerância a interferência de plantas competidoras e habilidade de supressão por cultivares de soja - I. Resposta de variáveis de crescimento. Planta Daninha, v. 23, n. 3, p. 405-414, 2005. https://doi.org/10.1590/S0100-83582005000300003

LONGCHAMPS, L., PANNETON, B., SIMARD, M. J., LEROUX, G. D. Could weed sensing in corn interrows result in efficient weed control? Weed Technol., v. 26, n. 4, p. 649-656, 2012.

https://doi.org/10.1614/WT-D-12-00030.1

LOGHAVI, M., MACKVANDI, B. B. Development of a target oriented weed control system. Computers and Electronics in Agriculture, v. 63, n. 2, p. 112-118, 2008. https://doi.org/10.1016/j.compag.2008.01.020

LUSCHEI, E. C., BUHLER, D. D., DEKKER, J. H. Effect of separating giant foxtail (Setaria faberi) seeds from soil using potassium carbonate and centrifugation on viability and germination. Weed Science, v. 46, n. 5 , p. 545-548, 1998. https://doi.org/10.1017/S0043174500091074

MOLIN, J.P., AMARAL, L.R., COLAÇO, A.F. Agricultura de Precisão. São Paulo: Oficina de Textos, 2015.

MONQUERO, P. A., CHRISTOFFOLETI, P. J. Banco de sementes de plantas daninhas e herbicidas como fator de seleção. Bragantia, v. 64, n. 2, p. 203-209, 2005. https://doi.org/10.1590/S0006-87052005000200006

MONQUERO, P. A., AMARAL, L. R., BINHA, D. P., SILVA, P. V., SILVA, A. C., MARTINS, F. R. A. Mapas de infestação de plantas daninhas em diferentes sistemas de colheita da cana-de-açúcar. Planta Daninha, v. 26, n. 1, p. 47-55, 2008. https://doi.org/10.1590/S0100-83582008000100005

PROCÓPIO, S. O., SILVA, A. A., SANTOS, J. B., FERREIRA, L. R., MIRANDA, G. V., SIQUEIRA, J. G. Efeito da irrigação inicial na profundidade de lixiviação do herbicida s-metolachlor em diferentes tipos de solos. Planta Daninha, v. 19, n. 3, p. 409-417, 2001. https://doi.org/10.1590/S0100-83582001000300014 
ROBERTSON, G.P. GS+: Geostatistics for the environmental sciences. Plainwell: Gamma Design Software, 1998.

TEIXEIRA, P. C., DONAGEMMA, G. K., FONTANA, A., TEIXEIRA, W. G. editores. Manual de métodos de análise de solo. Brasília, DF: Embrapa, 2017.

SCHAFFRATH, V.; TORMENA, C. A., GONÇALVES, A. C. A., OLIVEIRA JUNIOR, R. S. Variabilidade espacial de plantas daninhas em dois sistemas de manejo de solo. Revista Brasileira de Engenharia Agrícola e Ambiental, v. 11, n. 1, p. 53-60, 2007. https://doi.org/10.1590/S1415-43662007000100007

SILVA, A. F., CONCENÇO, G., ASPIAZÚ, I., FERREIRA, E. A., GALON, L., COELHO, A. T. C. P., SILVA, A. A., FERREIRA, F. A. Interferência de plantas daninhas em diferentes densidades no crescimento da soja. Planta Daninha, v. 27, n. 1, p. 75-84, 2009. https://doi.org/10.1590/S0100-83582009000100011

SILVA, F. M. L., CAVALIERI, S. D., SÃO JOSÉ, A. R., ULLOA, S. M., VELINI, E. D. Atividade residual de 2,4-D sobre a emergência de soja em solos com texturas distintas. Revista Brasileira de Herbicidas, v. 10, $\mathrm{n}$. 1, p. 29-36, 2011. https://doi.org/10.7824/rbh.v10i1.85

SHIRATSUCHI, L. S., FONTES, J. R. A., RESENDE, A. V. Correlação da distribuição espacial do banco de sementes de plantas daninhas com a fertilidade dos solos. Planta Daninha, v. 23, n. 3, p. 429-436, 2005. https://doi.org/10.1590/S0100-83582005000300006

SOARES, M. B. B., FINOTO, E. L., BOLONHEZI, D., CARREGA, W., ALBUQUERQUE, J. A. A., PIROTTA, M. Z. Fitossociologia de plantas daninhas sob diferentes sistemas de manejo de solo em áreas de reforma de cana crua. Agroambiente, v. 5, n. 3, p. 173-181, 2011. https://doi.org/10.18227/1982-

8470ragro.v5i3.594

STATISTICA. The Small Book Release 7.0. Tulsa: StatSoft Inc., 2004.

URI, N. D., ATWOOD, J. D., SANABRIA, J. The environmental benefits and costs of conservation tillage. Science of the Total Environment, v. 216, n. 1/2, p. 13-32, 1998. https://doi.org/10.1016/S00489697(98)00134-X

VASCONCELOS, M. C. C.; SILVA, A. F. A.; LIMA, R. S. Interferência de plantas daninhas sobre plantas cultivadas. Agropecuária Cientifica no Semi-Árido, v. 8, n. 1, p. 1-6, 2012.

VIEIRA, S.R. Geoestatística em estudos de variabilidade espacial do solo. In: Novais RF, Alvarez VH, Schaefer, CEGR, editores. Tópicos em ciência do solo. Viçosa: SBCS; 2000. p.1-54.

WARRICK, A.W., NIELSEN, D.R. Spatial variability of soil physical properties in the field. In: Hillel D, editor. Applications of soil physics. New York: Academic; 1980. p.319-344. https://doi.org/10.1016/B978-012-348580-9.50018-3 